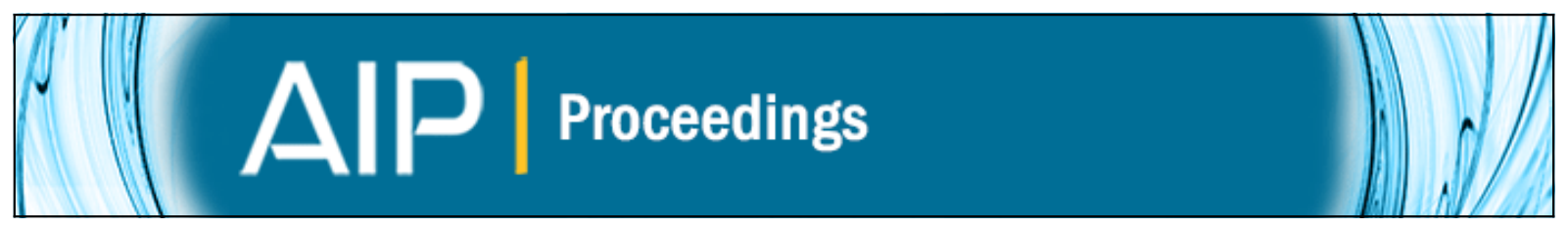

Spatiotemporal Response of Neuronal Networks to Time-Dependent Spectral Features

Pablo Balenzuela and Jordi García-Ojalvo

Citation: AIP Conference Proceedings 913, 178 (2007); doi: 10.1063/1.2746744

View online: http://dx.doi.org/10.1063/1.2746744

View Table of Contents: http://scitation.aip.org/content/aip/proceeding/aipcp/913?ver=pdfcov

Published by the AIP Publishing

Articles you may be interested in

Impact of delays on the synchronization transitions of modular neuronal networks with hybrid synapses

Chaos 23, 033121 (2013); 10.1063/1.4817607

Burst synchronization transitions in a neuronal network of subnetworks

Chaos 21, 016110 (2011); 10.1063/1.3559136

Delay-enhanced spatiotemporal order in coupled neuronal systems

Chaos 20, 043140 (2010); 10.1063/1.3528938

Spatiotemporal dynamics of networks of excitable nodes

Chaos 16, 015110 (2006); 10.1063/1.2177569

Dynamics of cultured neuronal networks

AIP Conf. Proc. 501, 240 (2000); 10.1063/1.59939 


\title{
Spatiotemporal Response of Neuronal Networks to Time-Dependent Spectral Features
}

\author{
Pablo Balenzuela* and Jordi García-Ojalvo ${ }^{\dagger}$ \\ ${ }^{*}$ Departamento de Física, Facultad de Ciencias Exactas y Naturales, Universidad de Buenos Aires, Pabellón 1, \\ Ciudad Universitaria (1428), Buenos Aires, Argentina \\ $\lceil$ Departament de Fisica i Enginyeria Nuclear, Universitat Politècnica de Catalunya, Colom 11, E-08222 \\ Terrassa, Spain
}

\begin{abstract}
In this work, we analyze the behavior of a neuronal network when it is stimulated by a synaptic train current with variable frequency. Using the fact that resonat (type II) neurons have a preferred input frequency, we constructed a network formed by two groups of neurons, each one responding to a different eigenfrequency. We show than even though every neuron is locally connected with neighbors of both groups, only those properly tuned to the input frequency fire synchronically with the incoming signal. In this sense, given a fixed sized network of heterogeneous neurons different subsets respond to the stimulus depending on its frequency.
\end{abstract}

Keywords: Episodic synchronization, neurons, brain networks, selective communication, resonance

PACS: 87.19.La, 05.45.Xt, 87.10.+e

\section{INTRODUCTION}

Brain networks have evolved to detect and process complex signals in a way that assure their survival. In recent years it has become clear that distinct pieces of information are not represented in the brain by a single dedicated neuron (the so-called grandmother neuron, which only fires when the observer sees her grandmother), but by populations of neurons that respond in a synchronized way to given signals [1]. One way in which this might be accomplished is by tuning different subsets of neurons to distinct input frequencies. In that way, a complex multicomponent signal could elicit the response of distinct collections of neuronal groups, which univocally represent the input signal. However, these different subsets of neurons will likely be densely interconnected and intermixed with each other, thus it remains to be seen if a combination of differently resonant neurons, thickly coupled to each other, is able to differentially respond to distinct input frequencies. This is not a trivial question, since coupling between neurons of different groups might in principle modify their response characteristics (including the location of the resonances), and this could elicit responses in unintended neurons.

To test the conjecture established above, we constructed a network of resonant (type II) neurons, consisting of two different groups that respond preferentially to different input frequencies. The response of isolated neurons to varying frequency inputs as a mechanism for selective communication was suggested by Izhikevich [2], and has been studied in detail in Ref. [3]. Previous studies in this direction were published by Segundo, Stiber and co-workers [4, 5]. The results shown below constitute an extension of the phenomenon to coupled networks of neurons.

\section{SIMULATIONS}

\section{Neuron model}

We consider neurons whose dynamical behavior is described by the Morris-Lecar model [6],

$$
\begin{aligned}
\frac{d V}{d t} & =\frac{1}{C_{m}}\left(I_{\mathrm{app}}-I_{\mathrm{ion}}-I_{\mathrm{syn}}\right) \\
\frac{d W}{d t} & =\phi \Lambda(V)\left[W_{\infty}(V)-W\right]
\end{aligned}
$$


where $V$ and $W$ represent the membrane potential and the fraction of open potasium channels, respectively. $C_{m}$ is the membrane capacitance per unit area and $\phi$ is the decay rate of $W$. The neuron is affected by several currents, including an external current $I_{\text {app }}$, a synaptic current $I_{\text {syn }}$, and an ionic current $I_{\text {ion }}$ given by

$$
I_{\text {ion }}=g_{C a} M_{\infty}(V)\left(V-V_{C a}^{0}\right)+g_{K} W\left(V-V_{K}^{0}\right)+g_{L}\left(V-V_{L}^{0}\right) .
$$

In this expression, $g_{a}(a=C a, K, L)$ are the conductances and $V_{a}^{0}$ the resting potentials of the calcium, potassium and leaking channels, respectively. We define the following functions of the membrane potential:

$$
\begin{aligned}
& M_{\infty}(V)=\frac{1}{2}\left[1+\tanh \left(\frac{V-V_{M 1}}{V_{M 2}}\right)\right] \\
& W_{\infty}(V)=\frac{1}{2}\left[1+\tanh \left(\frac{V-V_{W 1}}{V_{W 2}}\right)\right] \\
& \Lambda(V)=\cosh \left(\frac{V-V_{W 1}}{2 V_{W 2}}\right),
\end{aligned}
$$

where $V_{M 1}, V_{M 2}, V_{W 1}$ and $V_{W 2}$ are constants to be specified later. In the absence of noise, an isolated Morris-Lecar neuron shows a bifurcation to a limit cycle for increasing applied current $I_{\text {app }}$ [7]. Depending on the parameters, this bifurcation can be of the saddle-node or the subcritical Hopf types, corresponding to either type I or type II excitability, respectively. Type II neurons respond preferentially, in a resonant way, to certain non-zero input frequencies [3], and will be used in the network described below. The specific values of the parameters used are shown in Table 1 [8]. For these parameters, the threshold value of the applied current under constant stimulation is $46.8 \mu \mathrm{A} / \mathrm{cm}^{2}$ for type II.

\section{Synapsis model}

In this paper we analyze the behavior of a neuronal network driven by a synaptic current. To that end, we use the simplified model of chemical synapse proposed in Ref. [9], according to which the synaptic current is given by

$$
I_{\mathrm{syn}}=g_{\mathrm{syn}} r(t)\left(V-E_{S}\right)
$$

where $g_{\text {syn }}$ is the conductance of the synaptic channel, $r(t)$ represents the fraction of bound receptors, and $E_{s}$ is a parameter whose value determines the type of synapse: if $E_{S}$ is larger than the rest potential the synapse is excitatory, if smaller it is inhibitory; here we consider an excitatory synapse with $E_{s}=0 \mathrm{mV}$. The fraction of bound receptors, $r(t)$, follows the equation

$$
\frac{d r}{d t}=\alpha[T](1-r)-\beta r
$$

where $[T]=T_{\max } \theta\left(T_{0}+\tau_{\mathrm{syn}}-t\right) \theta\left(t-T_{0}\right)$ is the concentration of neurotransmitter released into the synaptic cleft by the presynaptic neuron. $\alpha$ and $\beta$ are rise and decay time constants, respectively, and $T_{0}$ is the time at which the presynaptic neuron fires, which happens whenever the presynaptic membrane potential exceeds a predetermined threshold value, in our case chosen to be $10 \mathrm{mV}$. This thresholding mechanism lies at the origin of the nonlinear character of the synaptic coupling. The time during which the synaptic connection is active is roughly given by $\tau_{\text {syn. }}$. The values of the coupling parameters that we use [9] are specified in Table 1. The equations were integrated using the Heun method [10], which is a second order Runge-Kutta algorithm for stochastic equations.

\section{NUMERICAL SETUP}

In a previous work [3] it was shown how a Morris-Lecar neuron responds to periodic synaptic inputs of varying frequency. The response of the neuron can be represented by a response diagram, showing the minimum excitation amplitude (represented by the synaptic coupling when we stimulate with synaptic currents) versus the frequency of the synaptic input. For type II neurons, the threshold input amplitude necessary to elicit spiking is minimum for a non-zero resonance frequency $f_{\text {res. }}$. This value is related with the frequency of the subthreshold oscillations, as analyzed in Ref. [3]. 
TABLE 1. Parameters values of the Morris-Lecar and synapse models used in this work.

\begin{tabular}{|c|c|c|c|}
\hline Parameter & Morris-Lecar TII & \multirow{4}{*}{ Parameter } & \multirow[b]{4}{*}{ Synapse } \\
\hline$C_{m}$ & $5 \mu \mathrm{F} / \mathrm{cm}^{2}$ & & \\
\hline$g_{K}$ & $8 \mathrm{mS} / \mathrm{cm}^{2}$ & & \\
\hline$g_{L}$ & $2 \mathrm{mS} / \mathrm{cm}^{2}$ & & \\
\hline$g_{C a}$ & $4.0 \mathrm{mS} / \mathrm{cm}^{2}$ & $\alpha$ & $2.0 \mathrm{~ms}^{-1} \mathrm{mM}^{-1}$ \\
\hline$V_{K}$ & $-80 \mathrm{mV}$ & $\beta$ & $1.0 \mathrm{~ms}^{-1}$ \\
\hline$V_{L}$ & $-60 \mathrm{mV}$ & $T_{\max }$ & $1.0 \mathrm{mM}$ \\
\hline$V_{C a}$ & $120 \mathrm{mV}$ & $g_{\text {syn }}$ & (specified in each case) \\
\hline$V_{M 1}$ & $-1.2 \mathrm{mV}$ & $\tau_{\text {syn }}$ & $1.5 \mathrm{~ms}$ \\
\hline$V_{M 2}$ & $18 \mathrm{mV}$ & $E_{S}$ & $0 \mathrm{mV}$ \\
\hline$V_{W 1}$ & $2 \mathrm{mV}$ & & \\
\hline$V_{W 2}$ & $17.4 \mathrm{mV}$ & & \\
\hline$\phi$ & $1 / 15 \mathrm{~ms}^{-1}$ & & \\
\hline
\end{tabular}

The frequency $f_{\text {res }}$ depends on the model parameters. In particular, in Morris-Lecar neurons, it is related with the dynamics of the slow variable $W$, and can be controlled by the parameter $\phi$. Figure 1 plots the response diagram for two different types of neurons. The results show that, depending on the value of the parameter $\phi$, the preferred spiking frequency $f_{\text {res }}$ takes different values. In this plot we only show the boundary of the 1:1 spiking region i.e., when the neuron spikes with the same frequency of input stimulus. We can see that depending on the strength of the synaptic coupling (or the amplitude of the synaptic current), we can tune the network in order that the spiking activity of one neuron would produce synchronous activity (or zero activity) on its neighbors neurons. This is the central idea of this paper: using the information given by the frequency dependent activity of one neuron, one can construct a network with global properties, such as for example, the ability of coding features spatially under certain input stimuli.

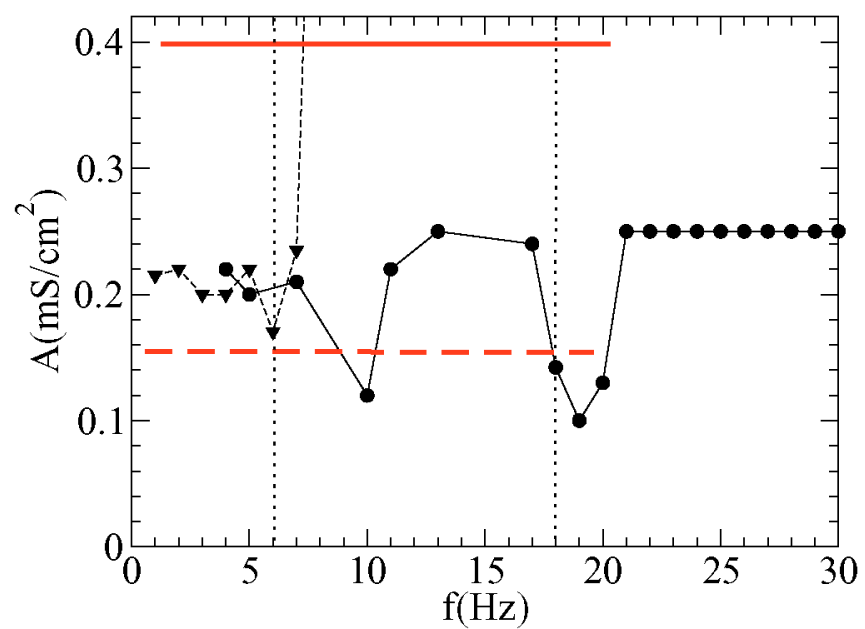

FIGURE 1. Response diagram for two different values of $\phi$, indicating the threshold above which the neuron responds in a $1: 1$ manner $\left(f_{\text {out }}=f_{\text {in }}\right)$. Triangles correspond to $\phi=1 / 55$; filled circles to $\phi=1 / 15$. Horizontal lines, both solid and dashed, denote the values of $g_{\text {syn }}$ used in the networks for the two groups of neurons, as indicated in Fig. 2. In vertical dotted lines, the frequency values of the input synaptic train.

Taking this into account, we constructed a square network with nearest neighbor coupling, containing two kinds of group neurons: one group (composing the number " 1 ") is characterized by a value of $\phi=1 / 55$, which leads to a resonance frequency $f_{\text {res }}=6 \mathrm{~Hz}$; the other group (composing the number "2") has $\phi=1 / 15$ and $f_{\text {res }}=18 \mathrm{~Hz}$. We choose three different values of the coupling strength: the neurons of group which form the number "1" are coupled 
to each other with a strength given by $g_{\text {syn }}=0.40 \mathrm{nS}$, the internal coupling among group cells forming number " 2 " has strength $g_{\text {syn }}=0.15 \mathrm{nS}$, and $g_{\text {syn }}=0.01 \mathrm{nS}$ for the rest of connections. A synaptic input train with two alternating frequencies, 6 and $18 \mathrm{~Hz}$, stimulates two neurons of the array. A scheme of this arrangement is shown in Fig. 2.

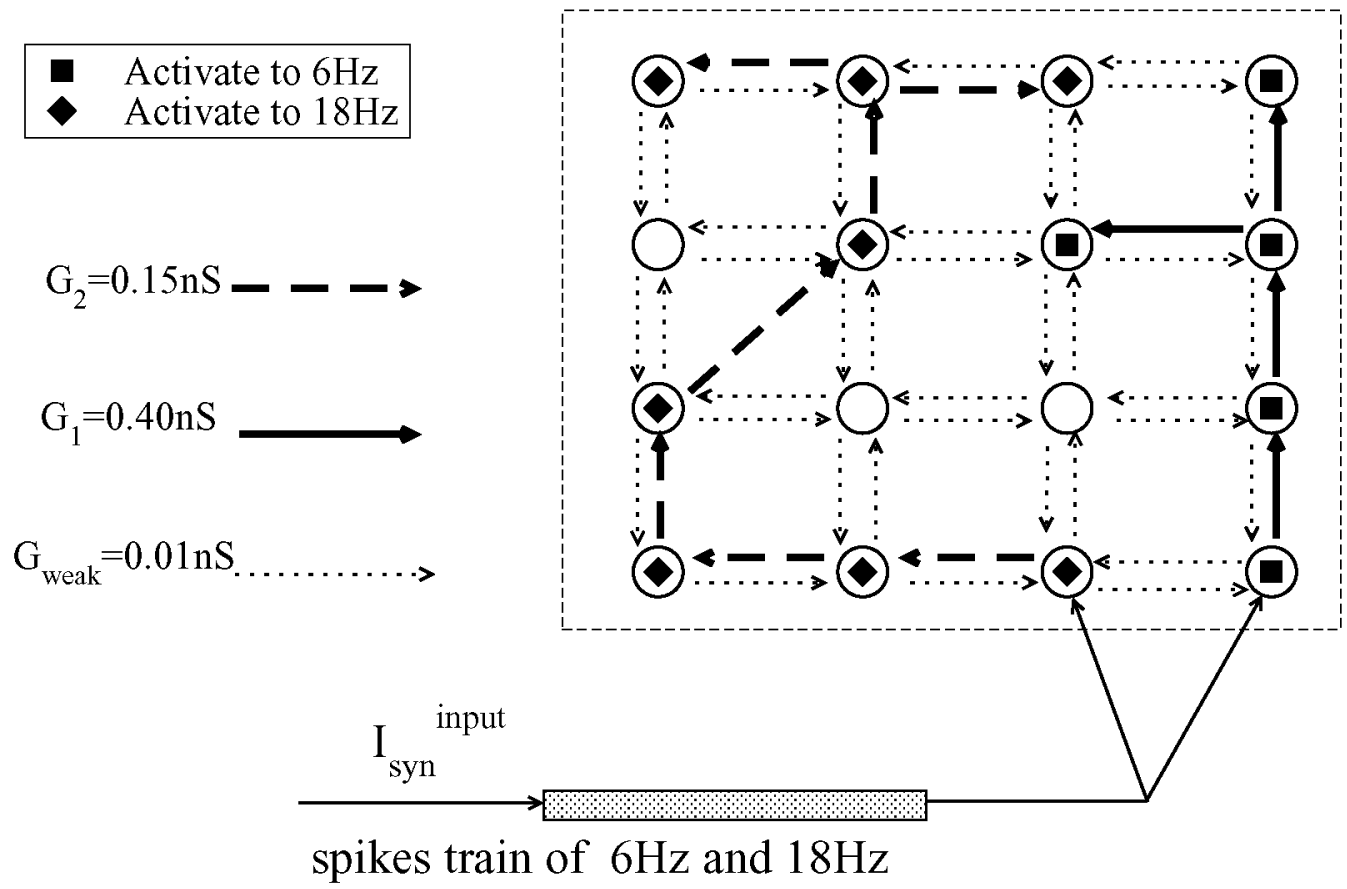

FIGURE 2. Scheme of the neuronal network studied: A $4 \times 4$ square lattice with nearest neighbors synaptic connection. Filled squares represent the neurons than fire at $6 \mathrm{~Hz}$, forming the number " 1 ". In black diamonds the neurons than fire at $12 \mathrm{~Hz}$, forming the number "2". Open circles represent the silent neurons. The stimulus (a synaptic train pulse with alternating 6 and $18 \mathrm{~Hz}$ frequencies) arrives to the down-right corner an the propagates along the network. The different values of $\phi$ of the group of neurons as well as the three different values of synaptic coupling $g_{\text {syn }}$ allow this setup to spell out the number " 1 " when the spike train is $6 \mathrm{~Hz}$ and "2" when is $18 \mathrm{~Hz}$.

The response of the array described above to the frequency-varying input is represented in Fig. 3, which shows the temporal series of a representative neuron of each group within the array. We can see how the response of the neurons alternates following precisely the variations in the input frequency, in such a way that a set of neurons only fire (synchronously) when the input frequency is $6 \mathrm{~Hz}$, and the other group fires when the input frequency is $18 \mathrm{~Hz}$. A similar selective response could be elicited for frequencies subharmonic of those two values considered here, provided the signal amplitude is large enough, as shown by the minima occurring at those frequencies in Fig. 1. This result illustrates that a fully connected network can host dynamical subnetwork activity in response to different types of input signals.

Finally, in order to illustrate the activity of the network taking into account the geometry of the array and the pattern generated by the subnetworks of synchronous neurons, we plot the activity of all the neurons in Fig. 4 in response to a given frequency varying synaptic current. We observe how the numbers " 1 " and " 2 " are formed alternatively in response to the different frequencies of the input.

\section{CONCLUSION}

Brain networks are subject to wide range of different signals, and mechanisms of selective communication should exist to discern between them. Taking advantage of the frequency selectivity of type II (resonant) neurons, we show that a locally coupled neuronal network composed of two intermixed groups of neurons, each one of them responding preferentially to a given input frequency, responds selectively to the input frequency, in such a way the each one of the 


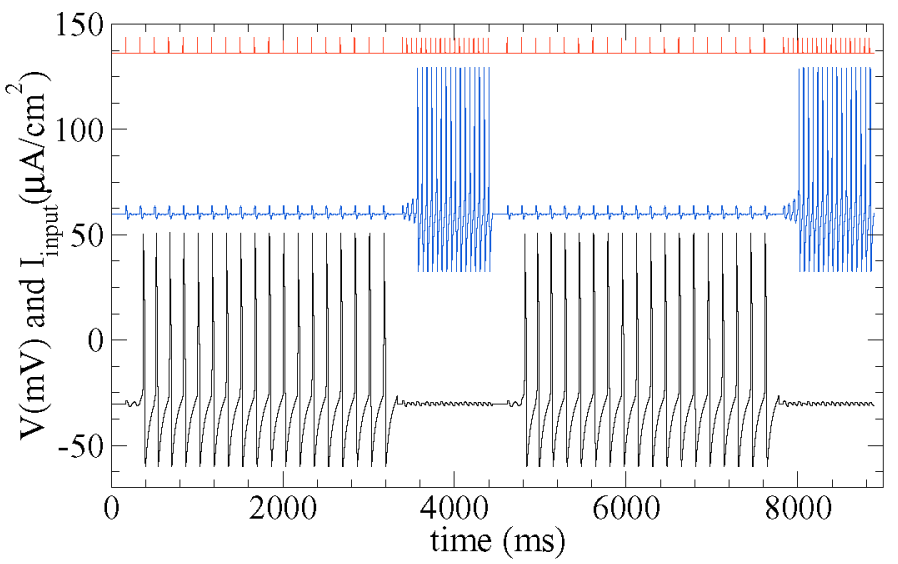

FIGURE 3. Time series of the two kind of neurons used in the array. Input current (top) and activity of the two group of neurons: Bottom, those that responds synchronously at $6 \mathrm{~Hz}$ and middle, those at $18 \mathrm{~Hz}$. We can observe that each group of neurons spikes only at the tuned frequency.

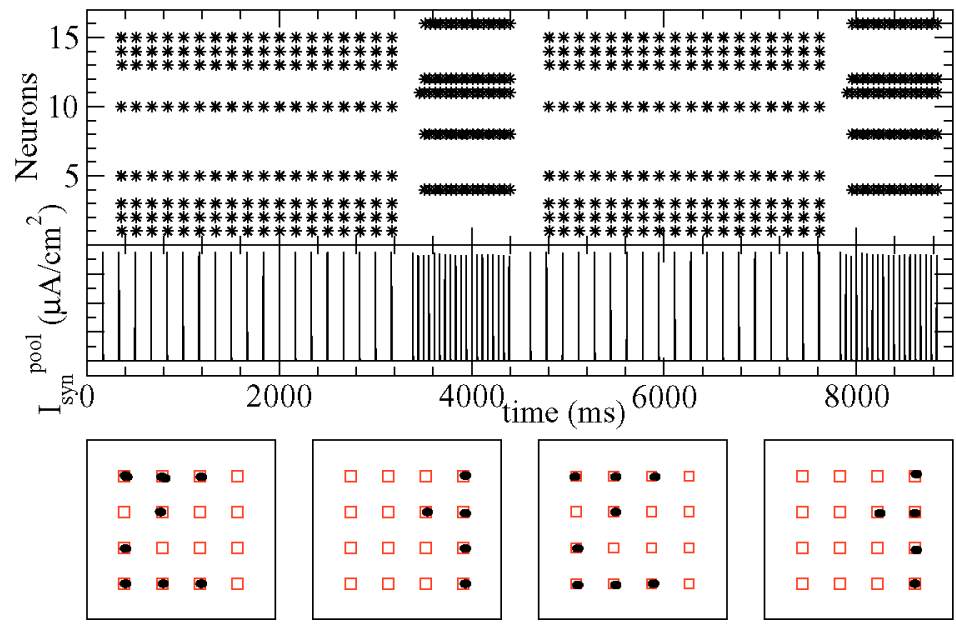

FIGURE 4. Spiking activity of the neuronal network, showing that two different subnetworks emerge dynamically in response to different input frequencies. Top panel: Raster plot showing the activity of the neurons and their dependence with the frequency of the input current showed in medium panel. Low panel: Activity of the network in the four characteristic stages showing how the numbers "1" and " 2 " are formed alternatively.

two groups is exclusively active when the input frequency coincides with the resonance of the corresponding group. The response of a single neuron to a varying frequency was studied in Ref. [3]. In that paper, the neuron was found to follow the input signal more or less adiabatically when the signal frequency entered the neuron's locking range for a given signal amplitude. Given those results, we expect that the neuronal network described here will act in the same way, with given subpopulations of neurons responding dynamically to that frequency to which they are resonant.

\section{ACKNOWLEDGMENTS}

We acknowledge financial support from the Ministerio de Educación y Ciencia (Spain, project FIS2006-11452), and from the Generalitat de Catalunya. P.B. acknowledges financial support from the Fundación Antorchas (Argentina), and from a C-RED grant of the Generalitat de Catalunya. P.B. is member of "Carrera de Conicet", Argentina. This work has also been supported by the European Commission (NEST project GABA, contract 043309). 


\section{REFERENCES}

1. W. Singer, Current Opinion Neurobiol. 9, 189-194 (1999).

2. E. M. Izhikevich, Biosystems 67, 95-102 (2002).

3. P. Balenzuela, J. M. Buldú, M. Casanova, and J. García-Ojalvo, Phys. Rev. E 74, 061910 (2006).

4. M. Stiber, R. Ieong, and J. P. Segundo, IEEE Trans. Neural Networks 8, 1379-1385 (1997).

5. J. P. Segundo, M. Stiber, J. F. Vibert, and S. Hanneton, Neuroscience 68, 693-719 (1995).

6. C. Morris, and H. Lecar, Biophys. J 35, 193-213 (1981).

7. M. St-Hilaire, and A. Longtin, J. Comp. Neurosci. 16, 299-313 (2004).

8. K. Tsumoto, H. Kitajima, T. Yoshinaga, K. Aihara, and H. Kawakami, Neurocomputing 69, 293-316 (2006).

9. A. Destexhe, Z. Mainen, and T. Sejnowski, Neural Comp. 6, 14-18 (1994).

10. J. García-Ojalvo, and J. M. Sancho, Noise in Spatially Extended Systems, Springer, New York, 1999. 